THE VI. EUROPEAN CONFERENCE ON CLINICAL HAEMORHEOLOGY

\title{
ORGANIZATION
}

I. VI. European Clinical Haemorheology Coordinating Committee

H. Lechner, E. Ott (Austria)

J.F. Stoltz, M.R. Boisseau (France)

T. Di Perri, o. Carandente (Italy)

J. Martins e Silva, P. de Souza Ramalho (Portugal)

M.W. Rampling, O.S. Roath (United Kingdom)

A.M. Ehrly, H. Schmid-Schönbein (W.Germany)

Corresponding Members:

Y. Isogai (Japan), H. Meiselman (USA)

II. Organizing Committee

A.M. Ehrly (Chairman)

H. Landgraf (Secretary/Treasurer)

J. Schenk

H. - G. Höffkes

K. Saeger-Lorenz

\section{Program Committee}

K. Breddin, Frankfurt; A.M. Ehrly, Frankfurt; P. Gaehtgens, Berlin; H. Hartert, Kaiserslautern; W. Heilmann, Essen; H. Kiesewetter, Homburg/Saar; H. Landgraf, Frankfurt; K. Messmer, Heidelberg; O. Linderkamp, Heidelberg; E. Volger, Bad Wörishofen; H. Schmid-Schönbein, Aachen.

IV. Patronage

Faculty of Medicine

Johann-Wolfgang-Goethe-University

Frankfurt am Main

German Society of

clinical Microcirculation and Haemorheology 